\title{
Corpos Anatomizados e Educação Médica: Identificando Intersecções entre Cultura, Formação e Prática Médica
}

\section{Anatomized Bodies and Medical Education: Identifying Intersections between Culture, Training and Medical Practice}

\author{
Vinícius Santos Rodrigues ${ }^{I}($ D \\ Cássia Beatriz Batista ${ }^{I}$ (iD \\ Marcelo Dalla Vecchia ${ }^{I}$ (DD
}

\section{PALAVRAS-CHAVE}

- Educação Médica.

- Cultura.

- Medicina.

- Anatomia.

Introdução: A prática médica é atravessada por um conjunto de ideais, valores e comportamentos compartilhados e transmitidos entre as especialidades e os diferentes perfis profissionais, exercendo influência significativa durante a graduação, especialmente por um currículo oculto. Método: Buscou-se levantar e caracterizar intersecções entre cultura, formação e prática médica no processo de anatomização do corpo. Foram realizadas entrevistas semiestruturadas com professores médicos de um curso de Medicina. Resultado: São apresentadas as discussões geradas a partir da análise do corpo anatomizado como habitus e suas repercussões para o entendimento da influência da cultura médica na formação. Conclusão: A anatomização do corpo humano está diretamente ligada a modelo de saúde biologicista, e a perspectiva da saúde integral depende de mudanças na forma de se encarar e ensinar o corpo. 


\section{KEYWORDS}

- Medical Education.

- Culture.

- Medicine.

- Anatomy.

Recebido em 20/6/20

Aceito em 22/6/20

\section{ABSTRACT}

Introduction: Medical practice is driven by a set of ideals, values, and behaviours shared and transmitted between specialties and different professional profiles, exerting significant influence during undergraduate training, especially by mean of a hidden curriculum. Method: We sought to identify and characterize the interactions between culture, medical education and medical practice in the process of body-anatomization. Semi-structured interviews were conducted with teachers at a medical school. Result: We discuss the analysis of the anatomized body as habitus, and its repercussions for the understanding of the influence of medical culture during undergraduate medical training. Conclusion: The anatomization of the human body is directly linked to the biological health model and the integral approach to health depends on changes in how we understand and teach students about the body.

\section{INTRODUÇÃO}

O debate sobre a cultura na sociedade contemporânea é central por possibilitar a estruturação e a organização de grupos sociais, uma vez que a força cultural se manifesta, organiza e desorganiza as diferentes categorias e lugares da sociedade ${ }^{1}$. Essa dinâmica influencia as manifestações e as práticas sociais, ao fazer com que as nossas ações passem a ser reguladas por um conjunto de normas e conhecimentos culturais, compartilhados por grupos específicos, constituindo subjetividades e criando identidades e diferenças dentro desses espaços ${ }^{2}$.

A cultura como institucionalizadora das ações sociais é a fonte de habitus na sociedade ${ }^{3}$. Embora haja contraposição de ideias e de aplicabilidade em relação ao habitus, segundo Pierre Bourdieu, o conceito emerge da necessidade empírica de apreender as relações de afinidade entre o comportamento dos agentes e das estruturas e os condicionamentos sociais ${ }^{4}$. Portanto, habitus é entendido como

[...] um sistema de disposições duráveis e transponíveis que, integrando todas as experiências passadas, funciona a cada momento como uma matriz de percepções, de apreciações e de ações - e torna possível a realização de tarefas infinitamente diferenciadas, graças às transferências analógicas de esquemas $[\ldots]^{3}$ (p. 65).

Em termos práticos, o habitus é concebido como princípio mediador e de correspondência entre as práticas individuais e as condições sociais ${ }^{4}$. Esse conceito adquire um alcance universal, por instrumentalizar o entendimento de sociedade, ao permitir examinar a coerência das características mais diversas de indivíduos submetidos às mesmas condições de existência ${ }^{5}$. O habitus pode ser concebido como um sistema de mediação entre o objetivo e o subjetivo, com disposições estruturadas e estruturantes, como ferramenta interpretativa da realidade de contextos sociais, funções e o modo de agir cotidiano na relação indivíduosociedade $^{6,7}$. O habitus da categoria médica é reforçado pelo paradigma flexneriano, que, dentro do modelo de ciência vigente, desenhou um ensino orientado pelas doenças, com ênfase na diferenciação entre o corpo normal e patológico, privilegiando a assistência hospitalar e a especialização da formação com uma perspectiva de trabalho liberal ${ }^{8-10}$.

No início da formação médica, o estudante busca adquirir informações e conciliar interesses pessoais e idealizações prévias sobre a profissão médica com as disciplinas ofertadas, constituindo idealizações de uma futura prática profissional baseada de modo central no aprendizado por modelos. Há uma necessidade por aprender medicina, em se tornar médico, que ao final do curso privilegia a aquisição e o acúmulo de informações ante uma aplicação imediata da prática, focada em diagnósticos, condutas e terapêuticas ${ }^{11}$. O que se percebe é uma mudança ao longo do curso das motivações de escolha e das percepções sobre o que é ser médico, dentro de um processo de modulação da identidade profissional, às vezes distante das expectativas iniciais do curso, por causa da própria dinâmica e exigência profissional ${ }^{11,12}$.

Esse percurso da formação se dá pela influência do imaginário social da profissão, pelas relações de poder e tradição e pelos benefícios sociais que ela pode gerar, o que ocorre antes do ingresso ao curso e sofre mudanças durante a graduação. $\mathrm{O}$ entendimento de como alguém se forma médico, portanto, vai além de conhecer a proposta curricular dos cursos de graduação e os conteúdos disciplinares ${ }^{13}$. É necessária uma compreensão mais ampla e complexa sobre o processo de aprendizagem que extrapola o prescrito nos planos de ensino e nos projetos pedagógicos. A compreensão desse fato exige uma diferenciação entre o que está sendo ensinado aos alunos e o que de fato eles estão aprendendo ${ }^{14}$. A busca pelo entendimento da formação médica, quando superado o conteúdo curricular formal, oportuniza um estudo sobre como esse profissional é compreendido socialmente, de como sua profissão foi socializada, organizada e estruturada e quem serão os novos médicos a perpetuar, ou a romper, com esse modo de produção cultural.

A profissão médica é composta por um conjunto de ideais, valores, linguagens e padrões comportamentais, inerentes a uma base técnica legítima e presentes em todas as especialidades; compartilhados e transmitidos entre os diversos perfis de profissionais desde os tempos de graduação ${ }^{15-17}$. O estudante, ao inserir-se em um contexto institucional, em contato com professores, médicos, residentes e outros profissionais da saúde, inicia-se na construção do seu "eu médico". A formação médica se dá por um modelo de médico a ser inspirado, seja pela demonstração de habilidades clínicas ou pela influência nas características profissionais ${ }^{14,18,19}$.

A graduação em Medicina é organizada por um currículo formal, paralelo e oculto ${ }^{20}$, e a lógica do aprendizado por modelo se manifesta nos diferentes tipos de currículo, especialmente no oculto que irá transformar em exemplos e inspirações aspectos sociais, culturais e subjetivos da profissão ${ }^{14}$. Entretanto, esses modelos não são neutros, e sim 
influenciados por diferentes aspectos sociais, de raça, de gênero, políticos e culturais ${ }^{21,22}$. Em uma graduação que privilegia a prática profissional na formação, o contato com os modelos de médicos ao longo do curso é fator de aprendizagem e conformação do habitus médico. Assim, o aluno seguirá as regras e os princípios regulatórios de um modelo bem organizado de formação, em que os conhecimentos, as experiências, as atitudes e os valores estarão sob a perspectiva de uma identidade médica em construção, atuando em diferentes situações, profissionais ou não ${ }^{23}$.

O currículo, dentro do pensamento crítico, representa uma seleção da cultura, uma escolha que se faz em um amplo universo, para assumir uma prática de significação, em meio a conflitos e disputas de poder, na produção de identidades sociais ${ }^{24}$. Galli ${ }^{25}$ afirma que o currículo oculto consiste na transmissão de uma cultura particular por meio de formas de identificação, em que o aluno incorpora a cultura médica, identifica os atributos de prestígio social e adquire valores. Esse conjunto de premissas se materializa na formação por meio do currículo oculto, em que a cultura médica influencia o comportamento e a prática a partir de construções do que é aceito para um ser médico ${ }^{26,27}$. Aspectos culturais do currículo oculto são passados pelo processo de ensino-aprendizagem e perpetuados por uma relação interpessoal entre professor-aluno ${ }^{26}$.

Nesse sentido, pensando em uma relação entre habitus e currículo oculto, o próprio perfil e a prática profissional de cada professor configuramse como importante fator de influência na formação ${ }^{28}$. Sistemicamente, essa relação é sujeita à interferência de todos os envolvidos no momento do aprendizado, abrangendo alunos, professores e instituições. Portanto, não há uma postura imparcial, supostamente à margem das ideologias, das influências ocultas da formação e daquilo que ocorre em outras dimensões da sociedade, como economia, cultura e política ${ }^{29}$. Assim, partimos da compreensão de que a educação médica é um processo cultural de influência de forças externas e internas à escola médica ${ }^{30}$.

A relação entre cultura, currículo e formação na constituição das identidades profissionais médicas nos leva a pensar que as identidades são construídas por meio da cultura, resultantes de uma complexa rede de aprendizagem de símbolos, códigos e padrões existentes nos grupos que as criaram. Esse fenômeno possibilita a manifestação de subjetividade entre seus membros, de modo que a cultura passa a ser mecanismo de influência do exterior, garantindo a existência de um grupo, e do interior, criando sentimento de pertencimento em seus membros, guiando e regulando práticas e existências pelo processo de socialização ${ }^{31}$. O contexto de formação médica, portanto, é fruto de uma intensa atuação da cultura médica, original e própria de seus pares, que atua como substrato na geração de médicos que buscam a legitimidade de suas respectivas identidades profissionais. Há nesse processo a apropriação e reprodução do habitus da categoria médica, em um contexto educacional mediado pelo currículo como objeto reprodutor de estruturas sociais ${ }^{32}$.

É relevante e estratégico discutir aspectos da cultura médica para ampliar e fundamentar debates em contextos de mudanças na formação ${ }^{14,26,27}$. Assim, em um cenário nacional de constantes reflexões e experimentações sobre mudanças dos paradigmas da formação médica ${ }^{33-36}$ e em saúde $e^{37}$, explorar os pontos compreendidos como centrais sobre a cultura médica inserida em um currículo oculto é ação imprescindível. A busca pela criação de novos significados e sentidos para o profissional médico é um desafio necessário e constante.

Em um estudo etnográfico do aprendizado médico, Bonet ${ }^{38}$ aponta a hierarquia do cuidado na saúde e a centralidade na doença e no diagnóstico como itens culturais da concepção médica biologicista e de uma prática biomédico-centrada, fruto de um processo de anatomização do corpo humano. Nessa direção, a prática médica se estabeleceu por um contato distante e frio com o corpo humano, reduzindo-o ao cadáver nas aulas de Anatomia. Assumir o corpo anatomizado como aspecto cultural de sistema de aquisição de habitus médico faz sentido para mapear locais de assimilação e manutenção dessa perspectiva de corpo. A partir dos ensinamentos da Antropologia, das Ciências Sociais e Educação Médica no Brasil, abordam-se neste artigo apontamentos de aspectos ligados à Anatomia Humana e aos corpos anatomizados como pontos de uma cultura médica incorporados dentro do currículo. Este estudo privilegia a perspectiva dos docentes, por serem agentes centrais na movimentação do currículo e na relação de aprendizagem da cultura médica, e nos permite aproximar de dimensões ocultas de um modelo de formação centrado no médico-professor.

A busca por intersecções entre corpos anatomizados aplicados à educação médica nos coloca perante os paradigmas do ensino da Anatomia. Ao longo do texto, será proposta uma discussão a partir das narrativas dos professores entrevistados em diálogo com autores que debatem cultura, formação e prática médicas.

\section{MÉTODOS}

O presente estudo contou com a participação dos professoresmédicos do corpo docente do curso de Medicina de uma universidade federal mineira: cerca de 50 profissionais, entre efetivos e substitutos, divididos nas grandes áreas médicas. Esta pesquisa de natureza exploratória buscou compreender, pela construção da identidade dos profissionais e das relações simbólicas que são atribuídas ao ser médico, quais aspectos culturais da categoria na relação com o habitus da prática médica podem estar envolvidos na formação médica e nas relações simbólicas da medicina.

Os dados foram coletados por entrevista semiestruturada com 12 professores-médicos. Todos os professores do departamento foram convidados a participar da pesquisa, sendo realizado convite formal em reunião departamental, no entanto os dados foram coletados dos professores que sinalizaram interesse e tinham disponibilidade para as entrevistas, conforme o cronograma para coleta de dados. Dos entrevistados, sete eram mulheres (52\%) e cinco homens (48\%), predominaram participantes entre 40 e 44 anos $(n=5,42 \%)$. Quanto ao tempo de conclusão da graduação, predominaram os participantes entre 11 e 20 anos $(n=5,43 \%)$. Sobre as especialidades, obtivemos respostas de representantes das seguintes áreas: medicina de família e comunidade, psiquiatria, cirurgia geral, clínica médica, neurologia, nefrologia, dermatologia e pediatria. As entrevistas tiveram em média duração de 20 minutos, realizadas durante o mês de dezembro de 2018 e em espaços diferentes: consultório particular, sala de atendimento no hospital, salas de aula e gabinetes da universidade.

Estabeleceu-se um roteiro que continha algumas questões centrais apoiadas nas hipóteses e teorias adotadas no estudo. Seguiu-se uma linha sócio-histórica, de cunho explicativo, com perguntas centrais que contemplavam aspectos como percepção e imaginário social do médico, mundo do trabalho, memórias do período de formação, críticas negativas e positivas ao ser médico, inspirações, fatores de influência para escolha

REVISTA BRASILEIRA DE EDUCAÇÃO MÉDICA

3 44(3) : e106; 2020 
profissional, limitações do exercício e motivações em continuar médico. A análise temática das entrevistas ${ }^{39}$ suscitou a delimitação de alguns focos de discussão pertinentes ao debate da cultura médica, como o que será destacado no item a seguir. Inicialmente, a anatomia e o corpo anatomizado não eram os protagonistas da discussão, mas a análise do material evidenciou que essas temáticas estão sujeitas à atribuição de valor e significado, direta ou indiretamente, no discurso dos professores, e o estudo desses pontos se demonstrou pouco explorado dentro do escopo central da pesquisa.

As entrevistas foram feitas de forma individual, em horário previamente agendado com os professores, sendo proposta a gravação do conteúdo, com a garantia do sigilo e a segurança do local de armazenamento. A pesquisa foi aprovada pelo Comitê de Ética em Pesquisa Envolvendo Seres Humanos das Unidades Educacionais de São João del-Rei (CEPSJ), Certificado de Apresentação para Apreciação Ética (CAAE) no 99243018.5.0000.5151, e todos os participantes, antes da coleta dos dados, assinaram o Termo de Consentimento Livre e Esclarecido (TCLE) em duas vias, entregando uma ao pesquisador-entrevistador.

\section{RESULTADOS}

O ensino da Anatomia no currículo médico inicia-se na Europa a partir do século XIV, na Universidade de Bolonha, marcado pela defesa da prática de dissecação de animais e seres humanos com o intuito de desenvolver pesquisas nas relações e nos paralelos existentes entre a vida humana e a animal ${ }^{40}$. $\mathrm{O}$ início dos estudos se constituiu pela prática descritiva das estruturas anatômicas, o que resultou na busca pelas relações funcionais das peças, bem como na incorporação dos ensinamentos por parte de outras disciplinas, como Fisiologia, e mais posteriormente Anatomia Patológica, na tentativa de se compreender organicamente o ser humano ${ }^{40}$. O caráter descritivo se demonstrou "estático" ante as complexidades envolvidas no estudo de cadáveres, já que não se revelavam as relações e implicações entre as estruturas identificadas com as dimensões da humanidade constituintes da vida, limitando-se a uma análise universal do normal e patológico.

A Anatomia como meio de investigação do corpo humano e de intervenção nele se consolidou como disciplina indispensável à formação em saúde, especialmente a médica ${ }^{41}$. Entretanto, há uma carência de discussões dos efeitos de um processo de ensino procedente de práticas laboratoriais, com cadáveres sem identidade, ainda focadas na dissecação e na memorização de peças e estruturas ${ }^{42}$. As propostas de inovação para o ensino-aprendizado de Anatomia limitam-se à inserção de novas metodologias de ensino durante as unidades curriculares ${ }^{43}$, mas ainda se observa uma tensão pela mudança, já que as bases fundantes da disciplina, como a fixação, a dissecação e a tradição, são de difícil mudança ${ }^{44}$ e estão distantes das complexidades envolvidas na formação médica. A urgência da mudança se dá pelo desempenho aquém do esperado e pelas altas taxas de reprovação, apesar das novas práticas e metodologias ${ }^{45}$, bem como pela necessidade de uma dimensão interdisciplinar na solidificação dos diversos campos teóricos necessários à formação médica, inexplorados no ensino anatômico tradicional ${ }^{46,47}$ :

Desde o começo, na Anatomia, aquele choque que a gente tem com cadáver... E eu quase fui reprovada em Anatomia e isso me marcou muito (Médica2).
A gente ficava fazendo dois anos de básico, que chamava disciplinas básicas. A Anatomia era quase um semestre e eu ficava de manhã, de tarde, até as dez horas da noite na sala de Anatomia, porque o médico precisa saber de Anatomia (Médico 12).

Aponta-se uma relação entre a exposição excessiva a conteúdos restritos aos conhecimentos anatomofisiológicos na formação do futuro profissional de saúde e um perfil profissional biomédico e medicalizador ${ }^{48}$. Foucault ${ }^{49}$, em sua complexidade, auxilia-nos na discussão aqui posta sobre o saber-poder da anatomia como prática discursiva no desenvolvimento, no domínio e na identificação do corpo patológico dentro do universo médico, marcado pela percepção do visível, de uma superfície capaz de manifestar o desenvolver da doença no corpo. Para Silva ${ }^{48}$,

O saber médico acaba por ser concebido e seu discurso enunciado como independente do corpo que os produzem, superiores ao restante da vivência humana, bem como preparados para se dirigirem a órgão e tecidos em si, absolutizados e isolado de um sistema mais amplo, de uma história pessoal, de uma cultura e de relações político-sociais [...] (p. 146).

A consolidação da prática médica com o objetivo de identificar a doença e propor a sua cura criou uma lógica de formação profissional excludente da subjetividade contida nos cadáveres estudados no início da graduação; há nesse ensino, o silêncio de vidas, raça, gênero e crenças. Essa negligência implica, no estudo anatômico, a atuação de um mecanismo dissociativo entre peças e corpos, doente e doença, e, futuramente, entre médicos e pacientes ${ }^{48}$. O ser humano passa a ganhar dimensões e formas, palpáveis e possíveis de estudos, pelo domínio dos corpos de outros seres humanos, possibilitando a manutenção do modelo de saúde focado na doença e no manejo dela ${ }^{38}$. Silva ${ }^{48}$ afirma que "[e]m nome da racionalidade científica moderna se exclui a subjetividade do adoecimento e relega-se o universo subjetivo do sofrer" (p. 148). Le Breton ${ }^{50}$, por sua vez, em seus estudos sobre a modernidade, constata que "[a] medicina moderna nasce dessa fratura ontológica, e a imagem que ela faz do corpo humano provém das representações anatômicas obtidas desses corpos sem vida, em que o homem não está presente" (p. 60).

[...] eu fiz a minha parte técnica do ensino médio em técnico de laboratório, então a gente usava os espaços da Faculdade de Medicina para fazer a parte técnica do curso, no ensino médio, $e$ aí eu comecei a conviver com aquele universo de entrar no anatômico de ver cadáver, de ver material de laboratório, e aquilo foi me despertando um pouco de interesse. E aí eu resolvi fazer Medicina! (Médico 1).

O meu interesse era a questão do humano, da pessoa... De poder saber mais sobre o ser humano... sobre o ser humano, sobre o corpo humano. [...] E aí na feira de ciências do sétimo período tinha o coração de um porco. E aí eu me lembro que eu vi o coração... Eles pegaram o coração do porco e dividiram ele em dois. Eu vi as câmaras. Eu olhei aquilo. Eu vi aquilo e fiquei tão fascinada... Foi muito... Eu fiquei hipnotizada, que eu fiquei aguardando todas as feiras de ciências até chegar no meu sétimo período [...] (Médica 5). 
A estruturação do corpo, formalizado em nível científico e biológico, como objeto necessário de estudo para a profissão médica, delimita disfarçadamente qual o propósito final dessa formação: o domínio e o controle desse corpo, orientados por princípios técnicos da Medicina. $\mathrm{O}$ ensino da Medicina funda-se, portanto, em uma análise entre o corpo biológico normal e o patológico, que exclui outras concepções de corpos, mas sendo esse objeto constituinte e intrínseco à prática médica:

Do centramento da medicina no corpo anátomo-fisiológico e na forma pela qual ele é conceitualizado no interior de um dado campo científico, decorre que o reconhecimento daquelas regularidades científicas construídas proporciona também o primeiro referencial para a elaboração dos conceitos de normal e patológico, inseparáveis da interferência técnica da medicina sobre o seu objeto. Esse referencial, todavia, não permite dar conta de todas as implicações subjacentes à normalidade ou de todas as determinações que se exercem sobre o corpo. O que implica afirmar, ao mesmo tempo, que o corpo como objeto da prática médica não se esgota em sua dimensão anátomofisiológica $^{51}$ (p. 23).

Ao deslocar a explicação das doenças e da morte da esfera da religiosidade, e ao assumir a condução dos fenômenos pelo aperfeiçoamento da clínica, o médico torna-se o responsável pelas respostas e explicações que cercam a complexidade humana ${ }^{52}$. O saber sobre o doente e sobre a doença é marcado no início do curso pela visualização e por práticas que manuseiam um corpo morto, dando início a um modelo de ser médico marcado pela negação dos significados e das interpretações sobre o corpo ${ }^{48}$. Nesse sentido, o aluno, ao lidar com o corpo morto, desenvolve maneiras de reprimir emoções e sentimentos e se distanciar da humanidade contida no cadáver, aprendendo pela relação professor-aluno-corpo não descrita no currículo, contudo presente na valoração de comportamentos "adequados" para um futuro médico. O ensino de Anatomia é, portanto, a inicialização do "eu médico" de cada aluno, em que uma concepção de corpo que se limita ao biológico é ensinada; é o momento em que se deixa de ser um estudante para tornar-se um médico em formação:

Eu tive uma grande dificuldade com aquela coisa de ter a formação dentro do hospital, aquela coisa de ver um paciente na enfermaria em vida e dois dias depois encontrar ele, como cadáver, lá na patologia clínica. Acabava comigo aquilo, era para mim extremamente adoecedor... [...] A gente vai ter que lidar em muitos momentos com cadáveres, com a morte... E, muitas vezes, de uma forma que a gente não vai estar totalmente preparado para aquilo, faz parte da sua formação como médica (Médico 4).

Outro aspecto relevante é a relação entre domínio e manuseio de peças anatômicas, já que o ensino de Anatomia com cadáver, sem nenhum envolvimento com as complexidades do binômio vida-morte, possibilita ao aluno experimentar a sensação de controle absoluto sobre o corpo, reproduzindo sentimento parecido no desenvolvimento da prática médica com os pacientes ${ }^{53}$. Aspectos desenvolvidos e manifestados durante o ensino da prática médica, como a relação de poder e controle sobre os pacientes e a centralidade da doença, do visível e do manifestado na superfície do corpo biológico, são itens necessários para repensar o processo de ensino-aprendizagem de Anatomia na área da saúde, especialmente na Medicina:

Quando a gente fala de Medicina, a primeira e mais clara discussão é a discussão do corpo, né? Não tem outro lugar... Eu me lembro de entrar em um museu da USP, museu de peças humanas e é uma coisa sem precedentes. É uma coisa enorme. São estantes e estantes de partes humanas, e eu me lembro que eu não fiquei nem um pouco traumatizada. Eu fiquei fascinada, e a minha tia que foi comigo ficou enlouquecida [...] Eu fiquei tão encantada com o "anatômico" e para outras pessoas foram traumáticas, mas eu gostava dessa questão [...] (Médica 5).

\section{DISCUSSÃO}

Como pensar em uma aula de Anatomia que supere a concepção corpo-centrada, que amplie a noção de corpo, que traga uma abordagem das narrativas construídas por esse cadáver de maneira a permitir uma reflexão sobre os aspectos da humanidade ali manifestados? E qual é o propósito de ensino da Anatomia nos currículos médicos de hoje?

$\mathrm{O}$ atual modelo de ensino da Anatomia marca um processo de divisão de sujeitos e de seus respectivos corpos que, por conseguinte, corrobora a fragmentação da perspectiva de cuidado integral à saúde. O corpo anatomizado como aspecto cultural predomina no discurso médico, e o controle do corpo ou mesmo da vida ainda orienta a formação para uma identidade médica biomédica. Existe entre os entrevistados a ideia de que a aprendizagem do corpo anatomizado é imprescindível para o ser médico. Nesse sentido, quando se pensa a Anatomia como aspecto cultural da Medicina, é possível traçar paralelos entre a inicialização do habitus médico e a exposição a uma concepção puramente biológica sobre corpos, saúde e cuidado. O habitus médico se inicia em locais estratégicos de aquisição e manifestação de cultura. Assumir a existência de uma cultura que fragmenta o ser humano e o cuidado dentro de um currículo formal e oculto e que estrutura um habitus próprio da Medicina é um passo importante para reflexões e mudanças da formação das identidades médicas.

O ensino da Anatomia fragmentado e descontextualizado em relação aos outros saberes em saúde parece ser contrário aos próprios anseios de um profissional crítico, reflexivo e humanístico. O professor, em seu compromisso de mediar o aprendizado, torna-se responsável por desenvolver reflexões sobre ética e relação vida-morte, doença-corpo e médico-paciente existentes em um contexto de ensino anatômico. Freire ${ }^{54}$ sinaliza que o ato de ensinar é espaço para pensar, em que é fundamental a reflexão crítica contínua sobre a prática. Os envolvidos com o ensinoaprendizagem da anatomia devem repensar o processo educacional e estruturá-lo de modo a oportunizar mudanças de pensamentos, ações e condutas ${ }^{55}$ sobre o significado do corpo humano. A reorientação do ensino anatômico deve caminhar para a construção de significados e de proposições de intervenções na realidade dos envolvidos, na construção do saber em saúde ${ }^{56}$. Qual é, portanto, o significado de uma aula de Anatomia que se mantém inerte ante as mudanças? Ainda, quais são as transformações geradas em uma aula de Anatomia que se mantém inerte ante as mudanças? Saídas para a resolução desse dilema passam pela recuperação do caráter indissociável e integral da prática docente, em contraponto à fragmentação do ensino e do conhecimento ${ }^{57}$.

Boa parte dos entrevistados destaca a centralidade do corpo como

$\mid$ REVISTA BRASILEIRA DE EDUCAÇÃo MÉDICA

5 44 (3) : e106; 2020 
ponto importante para pensar identidades médicas, por vezes, sem explorar as tensões que as diferentes concepções sobre corpos provocam para a saúde. O corpo é espaço de análise de sujeitos, pois, a partir desse objeto, é possível captar o ser humano histórico, determinado socialmente e imerso em um universo. Esse objeto, que vai além do biológico, dá consistência ao nosso imaginário social e é espaço de inscrição da condenação social, em que se leem as regras familiares, de classe e de limitações coletivas. O corpo humano é um sistema afetado pela religião, pela ocupação, pelo grupo familiar, pela classe, pela raça e por diversos outros intervenientes sociais e culturais ${ }^{58}$. Na saúde, compreender o corpo implica estabelecer relações entre sujeito, natureza e cultura, uma vez que o ser humano é fruto desses polos e sofre influência de valores, crenças, política e economia ${ }^{59}$, além de possibilitar acesso à estrutura social vigente $^{60}$. Mudanças na formação passam pela reordenação do ensino, pela atitude atenta aos aspectos culturais da anatomia e pela postura inovadora com o conteúdo programático, flexível com a carga horária das aulas e contrária à tradição sobre o ensino da Anatomia ${ }^{61}$.

\section{CONCLUSÃO}

O corpo anatomizado como conhecemos e suas repercussões na formação médica não cabem às necessidades de saúde. A reprodução de uma perspectiva biologicista do corpo no centro da formação médica se distancia do perfil do egresso necessário. A percepção da influência do processo de anatomização do corpo na produção de discursos do ser médico evidencia a necessidade de criar mecanismos para ampliar a compreensão da influência do habitus da prática médica em um currículo.

A formação em saúde e para o Sistema Único de Saúde (SUS) almeja que o ensino sobre o corpo vivo, histórico e social suscite discussões e reflexões sobre o objeto de ensino e sobre as práticas pedagógicas. Convocamos aqui a Anatomia para um papel de interceptora de inúmeras discussões em saúde necessárias à formação médica, de forma interdisciplinar e socialmente relevante, que se contraponha aos currículos de conteúdos tradicionais.

A reformulação do ensino de Anatomia com foco na construção de um saber integral, com debate em torno das diversas disciplinas que abordam o corpo humano e em diálogo com a saúde e a cultura, é um grande desafio aos docentes. Entretanto, uma discussão interdisciplinar e compartilhada entre os núcleos da saúde talvez seja o propósito atual para as aulas de Anatomia. O ensino da Anatomia Humana, portanto, está diretamente ligado ao modelo de saúde biologicista, e a garantia de um modelo de saúde integral depende, assim, de mudanças na forma de encarar e ensinar o corpo. A formação médica é um espaço de aquisição de cultura, e uma nova cultura médica sobre o corpo necessita ser iniciada.

\section{AGRADECIMENTO}

Ao Programa Institucional de Bolsas de Iniciação Científica da Universidade Federal São João del-Rei - UFS (Pibic/CNPq/UFSJ).

\section{REFERÊNCIAS}

1. Hall S. A centralidade da cultura: notas sobre as revoluções culturais do nosso tempo. Educ Real. 1997;22(2):15-46.

2. Ferrari MALD. O papel da diferença na construção da identidade. Bol Psicol. 2006;56(124):1-8.

3. Ortiz R. Bourdieu: sociologia. São Paulo: Ática; 1983.
4. Setton MGJ. A teoria do habitus em Pierre Bourdieu: uma leitura contemporânea. Rev Bras Educ. 2002;20:60-70.

5. Pinto L. Pierre Bourdieu e a teoria do mundo social. Rio de Janeiro: Editora da FGV; 2000.

6. Wacquant L. Seguindo Pierre Bourdieu no campo. Rev Sociol Polit. 2006;26:13-29.

7. Araújo CM, Oliveira MCSL. Contribuições de Bourdieu ao tema do desenvolvimento adolescente em contexto institucional socioeducativo. Pesqui Prát Psicossociais. 2014;8(2):215-24.

8. Veloso TCMA. Programa Nacional de Incentivo a Mudanças Curriculares no Curso de Medicina: a experiência da UFG [tese]. Goiânia: Universidade Federal de Goiás; 2008.

9. Machado $\mathrm{M}$, coordenadora. Os médicos no Brasil: um retrato da realidade. Rio de Janeiro: Editora Fiocruz; 1997.

10. Rezende JM. À sombra do plátano: crônicas de história da medicina. São Paulo: Editora Unifesp; 2009.

11. Dini PS, Batista NA. Graduação e prática médica: expectativas e concepções de estudantes de Medicina do $1^{\circ}$ ao $6^{\circ}$ ano. Rev Bras Educ Med. 2004;28(3):198-203.

12. Oliveira RZ, Gonçalves MB, Bellini LM. Acadêmicos de medicina e suas concepções sobre "ser médico". Rev Bras Educ Med. 2011;35(3):311-8.

13. Lampert JB. Tendências de mudanças na formação médica no Brasil: tipologia das escolas. São Paulo: Hucitec; 2002.

14. Hafferry FW. Beyond curriculum reform: confronting medicine's hidden curriculum. Acad Med.1998;73(4):403-7.

15. Rego SO. O processo de socialização profissional na medicina. In: Machado MH, organizadora. Profissões de saúde: uma abordagem sociológica. Rio de Janeiro: Fiocruz; 1995.

16. Machado MH. Sociologia das profissões: uma contribuição ao debate teórico. In: Machado MH, organizadora. Profissões de saúde: uma abordagem sociológica. Rio de Janeiro: Fiocruz; 1995.

17. Pereira-Neto AF. A profissão médica em questão (1922): dimensão histórica e sociológica. Cad Saúde Pública. 1995;11(4);1-16.

18. Irby DM. Clinical teaching and the clinical teacher. J Med Educ. 1986;61(9):35-45.

19. Moroz M, Luna, SV. Professor: o profissional do ensino! Reflexões do ponto de vista behaviorista/comportamental. Psicol Educ. 2013;36:115-21.

20. Rego S. Parallel curriculum in Medicine, clinical practice, and Problem Based Learning: is there a way out? Interface. 1998;2(3):35-48.

21. Wright SM, Carrese JA. Serving as a physician role model for a diverse population of medical learners. Acad Med. 2003;78(6):623-8.

22. McLean M. Is culture important in the choice of role models? Experiences from a culturally diverse medical school. Med Teach. 2004;26(2);142-9.

23. Gomes AP, Rego S. Pierre Bourdieu and medical education. Rev Bras Educ Med. 2013;37(2): 260-5.

24. Moreira AFB. Currículo, cultura e formação de professores. Educ Rev. 2001;(17):39-52.

25. Galli A. Argentina: transformación curricular. Educ Méd Salud. 1989;23(4);344-53.

26. Haidet Paul, Howard FS. The role of the student-teacher relationship in the formation of physicians. The hidden curriculum as process. J Gen Intern Med. 2006;21(S1):S16-S20. 
27. Passi V, Johnson S, Peile E, Wright S, Hafferty F, Johnson N. Doctor role modelling in medical education: BEME Guide $\mathrm{n}^{\circ}$. 27. Med Teach. 2013 Sep;35(9):e1422-36.

28. Cruess RS, Cruess LR, Steinert Y. Role modelling - making the most of a powerful teaching strategy. BMJ. 2008;336(7646):718-21.

29. Haddad AE, Morita MC, Pierantoni CR, Brenelli SL, Passarella T, Campos FE. Formação de profissionais de saúde no Brasil: uma análise no período de 1991 a 2008. Rev Saúde Pública. 2010;44(3):383-93.

30. Schein EH. Organizational culture and leadership. San Francisco, CA: Jossey-Bas; 1992.

31. Setton MGJ. Processos de socialização, práticas de cultura e legitimidade cultural. Estud Sociol. 2010;15(28):18-35.

32. Silva TT. Documentos de identidade: uma introdução às teorias do currículo. Belo Horizonte: Autêntica; 2005.

33. Feuerwerker LCM. Além do discurso de mudança na educação médica: processos e resultados. São Paulo: Hucitec; 2002.

34. Lampert JB, Costa NMSC, Perim GL, Abdalla IG, Aguilar-da-Silva RH, Stella RCR. Tendências de mudanças em um grupo de escolas médicas brasileiras. Rev Bras Educ Med. 2009;33(1):19-34.

35. Nogueira MI. As mudanças na educação médica brasileira em perspectiva: reflexões sobre a emergência de um novo estilo de pensamento. Rev Bras Educ Med. 2009;33(2):262-70.

36. Oliveira FP. As mudanças na formação médica introduzidas pelo Programa Mais Médicos [tese]. Brasília: Universidade de Brasília; 2018.

37. Batista CB. Movimentos de reorientação da formação em saúde e as iniciativas ministeriais para as universidades. Barbarói. 2013;38(1):97-125.

38. Bonet $O$. Saber e sentir: uma etnografia da aprendizagem da biomedicina. Rio de Janeiro: Fiocruz; 2004.

39. Braun V, Clarke V. Using thematic analysis in psychology. Qual Res Psychol. 2006;3(2):77-101.

40. Talamoni AB, Bertolli Filho C. A anatomia e o ensino de anatomia no Brasil: a escola boveriana. Hist Ciênc Saúde-Manguinhos. 2014;21(4):1301-22.

41. Turney BW. Anatomy in a modern medical curriculum. Ann R Coll Surg Engl. 2007;89(2):104-7.

42. Fornaziero CC, Gil CRR. Novas tecnologias aplicadas ao ensino da anatomia humana. Rev Bras Educ Med. 2003;27(2):141-6.

43. Reis C, Martins MM, Mendes RAF, Gonçalves LB, Sampaio Filho HC, Morais MR, et al. Avaliação da percepção de discentes do curso médico acerca do estudo anatômico. Rev Bras Educ Med. 2013;37(3):350-8.

44. Tavano PT, Almeida MID. A reconfiguração do ensino anatômico: tensões que incidem na disciplina básica. Rev Bras Educ Med. 2011;35(3):421-8.

45. Silva JH, de Sá MA, Schetino LPL, Guerra LB. O ensino-aprendizagem da anatomia humana: avaliação do desempenho dos alunos após a utilização de mapas conceituais como uma estratégia pedagógica. Ciênc Educ (Bauru). 2018;24(1):95-110.

46. Kluchova D. New pedagogic methods in anatomy: experience at Cambridge University. Bratisl Lek Listy. 2000;101(1):58-60.
47. Johnson EO, Charchanti AV, Troupis TG. Modernization of ananatomy class: from conceptualization to implementation: a case for integrated multimodal - multidisciplinary teaching. Anat Sci Educ. 2012;5(6):354-66.

48. Silva GSN. A construção do "ser médico" e a morte: significados e implicações para a humanização do cuidado [tese]. São Paulo: Universidade de São Paulo; 2007.

49. Foucault M. O nascimento da clínica. Rio de Janeiro: Forense Universitária, 1987.

50. Le Breton D. Anthropologie du corps et modernité. Paris: PUF; 1995.

51. Donnangelo MCF. Saúde e sociedade. São Paulo: Duas Cidades; 1976.

52. Sayd JD. A escola médica e seus implícitos sobre a morte. Rev Bras Educ Med. 1993; 17(3):14-20.

53. Zaidhaft S. Morte e formação médica. Rio de Janeiro: Francisco Alves; 1990.

54. Freire P. Pedagogia da autonomia: saberes necessários a prática educativa. São Paulo: Paz e Terra; 1996.

55. Freitas DA, Santos EMS, Lima LVS, Miranda LN, Vasconcelos EL, Nagliate PC. Teachers' knowledge about teaching-learning process and its importance for professional education in health. Interface (Botucatu). 2016;20(57):437-48.

56. Batista NA. Desenvolvimento docente na área da saúde: uma análise. Trab Educ Saúde. 2005;3(2):283-94.

57. Saviani D. Formação de professores: aspectos históricos e teóricos do problema no contexto brasileiro. Rev Bras Educ. 2009;14(40):143-55

58. Montagner MA. Pierre Bourdieu, o corpo e a saúde: algumas possibilidades teóricas. Ciênc. Saúde Colet. 2006;11(2):515-26.

59. Polak YNS. A concepção de corpo no mundo da saúde. Cogitare Enfer. 1996;1(1):4-9.

60. Rodrigues JC. Tabu do corpo. Rio de Janeiro: Fiocruz; 2006.

61. Drake RL. Anatomy education in a changing medical curriculum. Anat Rec. 1998;253(1):28-31.

\section{CONTRIBUIÇÃO DOS AUTORES}

Vinícius Santos Rodrigues concebeu o projeto e atuou na obtenção de dados. Vinícius Santos Rodrigues e Cássia Beatriz Batista elaboraram o projeto de pesquisa. Vinícius Santos Rodrigues, Cássia Beatriz Batista e Marcelo Dalla Vecchia realizaram a análise e interpretação de dados, elaboraram o esboço do artigo, fizeram a revisão crítica e aprovaram a versão final a ser publicada.

\section{CONFLITO DE INTERESSES}

Os autores declaram não haver conflito de interesses neste estudo.

\section{ENDERECO PARA CORRESPONDÊNCIA}

Vinícius Santos Rodrigues. Rua Castelo Novo, 82, Santa Efigênia, Belo Horizonte, MG, Brasil. CEP: 30260-380.

E-mail: viniciushp95@gmail.com 